Utah State University

DigitalCommons@USU

\title{
$5-2019$
}

\section{Staying Upright by Shutting Down? Evidence for Global Suppression of the Motor System When Recovering Balance}

\author{
Caleigh Goode \\ Utah State University
}

Follow this and additional works at: https://digitalcommons.usu.edu/gradreports

Part of the Kinesiology Commons

\section{Recommended Citation}

Goode, Caleigh, "Staying Upright by Shutting Down? Evidence for Global Suppression of the Motor System When Recovering Balance" (2019). All Graduate Plan B and other Reports. 1359.

https://digitalcommons.usu.edu/gradreports/1359

This Creative Project is brought to you for free and open access by the Graduate Studies at DigitalCommons@USU. It has been accepted for inclusion in All Graduate Plan B and other Reports by an authorized administrator of DigitalCommons@USU. For more information, please contact digitalcommons@usu.edu.

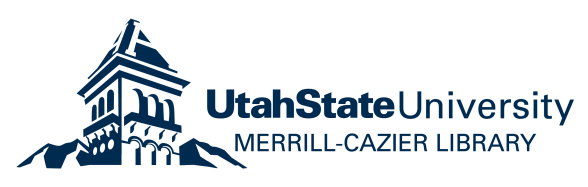


STAYING UPRIGHT BY SHUTTING DOWN? EVIDENCE FOR GLOBAL

SUPPRESSION OF THE MOTOR SYSTEM WHEN RECOVERING BALANCE

by

Caleigh Goode, LAT, ATC

A plan B research project submitted in partial fulfillment

of the requirements for the degree

of

MASTER OF SCIENCE

in

Health and Human Movement

Approved:

David Bolton, Ph.D.

Major Professor
Chris Dakin, Ph.D.

Committee Member

Eadric Bressel, Ph.D.

Committee Member

UTAH STATE UNIVERSITY

Logan, Utah 
Copyright (C) Caleigh Goode 2019

All Rights Reserved 


\begin{abstract}
Background: When automatic, yet unwanted action is quickly inhibited, short-lived suppression throughout the motor system ensues. This type of response inhibition causing an all-over motor suppression effect is referred to as global suppression. Although response inhibition is essential for behavioral flexibility, widespread motor suppression may delay action reprogramming. In reactive balance control, even fleeting suppression of the motor system could interfere with our ability to adapt compensatory reactions quickly enough to avoid a fall. Whether this concept of global suppression is occurring in situations that pose a postural threat is undetermined.
\end{abstract}

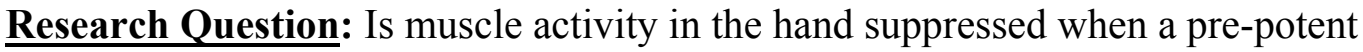
compensatory step becomes suddenly blocked in a balance recovery task?

Methods: Nineteen young adults were tested using a lean and release apparatus. Participants were occasionally released from a support cable resulting in forward body displacement. At the start of each trial, vision was occluded and a leg block was either placed in front of the legs or removed to allow a forward step. After goggles opened, the cable was released to cause a postural perturbation and participants had to either quickly step forward (STEP) or use a feet-in-place reaction to regain stability (NO-STEP). Step trials were much more frequent to promote stepping. Transcranial magnetic stimulation (TMS) was delivered shortly after receving vision (but before postural perturbation) to assess corticospinal excitability in an intrinsic hand muscle that was irrelevant to the balance recovery task. 
Results: Repeated measures ANOVA compared motor-evoked potentials across two step conditions (STEP, NO-STEP) and two TMS latencies (100ms, 200ms). The time x step condition interaction was significant with $(F(1,18)=4.47, \mathrm{p}=0.049)$. The resultant interaction provided evidence of motor suppression in the hand when a forward step was blocked.

Significance: Inhibition of a hand muscle uninvolved in a compensatory leg response provided evidence of global suppression in a whole-body, reactive balance context. Such widespread suppression of the motor system has implications for maintaining postural equilibrium, where even a momentary shutdown across body regions could interfere with the ability to adapt corrective balance reactions. 


\section{Introduction}

Many situations in daily life include unpredictable environments that require an action response that is both quick and appropriate. Oftentimes, an automated response must be suppressed in favor of a more appropriate reaction. Reactive balance control is an important example in which case actions need to be extremely fast in order to avoid a fall, while simultaneously avoiding obstacles in the environment. Oftentimes, spinal and brain stem reflexes are insufficient to meet the demands of behavioral flexibility to adapt to complex or changing environments. While numerous studies now attest to a correlation between higher brain function and balance (Herman, Mirelman, Giladi, Schweiger, \& Hausdorff, 2010; Mirelman et al., 2012; Muir, Gopaul, \& Montero Odasso, 2012), little is known about the underlying mechanisms (Bolton, 2015). Falls are the leading cause of fatal and nonfatal injuries in adults over the age of 65 (Bergen, Stevens, \& Burns, 2016), which emphasizes the need to understand how we control balance at a higher level of brain function to determine what particular deficits lead to a fall. The ability to suppress a pre-potent action and selecting more suitable reactions is operated by this higher level of brain functioning. The quick change in action requires inhibition of an automatic response (i.e. a recovery step), in a process called response inhibition. While this is a fundamental aspect of neural control of behavior, it also has great implications for balance control.

\section{Response inhibition and its role in reactive balance}

Various situations in everyday life cause us to adapt actions in order to avoid falls. The cognitive ability to stop and adapt actions relevant to the presented environment for balance recovery is a key higher functioning brain mechanism that may contribute to fall 
prevention. For example, passengers on a moving bus that comes to a sudden stop must respond quickly to avoid a fall. If the bus is not too crowded, the passenger's first reaction will be to change their base of support by taking a quick step to recover their balance. Generating a well-placed step with sufficient speed are hallmarks of an effective compensatory stepping response (Rogers \& Mille, 2016). If the bus is crowded, however, and there are bags or groceries on the floor of the bus, the passenger cannot take a forward step and may need to supress this action and initiate a more appropriate action such as maintaining a fixed support or grabbing onto a handle. Some situations require a change in support reaction, such as shifting balance to the other leg or grabbing onto a handrail, to recover balance with larger perturbations or by maintaining balance in one leg while the other leg navigates an obstacle for a well-placed recovery step (Maki \& McIlroy, 2007). The brain needs to quickly assess the environment and turn on the appropriate response while suppressing an unwanted action, such as stepping into an obstacle. An automated step may not be the appropriate response and can cause greater postural threat in situations such as a stairwell or where there is a more effective alternative present (i.e. grabbing onto a more supportive foundation such as a handrail). This response inhibition could occur because there needs to be an appropriate behavioural override.

\section{Global inhibition}

There are thought to be two main mechanisms of response inhibition, selective inhibition and global inhibition. The ability to shut off one response but continue another action requires selective inhibition (Aron \& Verbruggen, 2008). Global inhibition 
however rapidly shuts down all ongoing behavior both in terms of cognition and motor function (Aron \& Verbruggen, 2008). Motor inhibition occurs when a current ongoing action needs to be suppressed following some type of stop signal. This is often a fast and global suppression - one way in which it can be quantified is as a decrease in corticospinal excitability - in both task relevant and task irrelevant muscles to generically brake motor output (Aron \& Verbruggen, 2008). A global suppression of motor output represents a broad shutdown of motor activity throughout the whole body. Often times, this kind of stop signal in real life is in the form of an unexpected event. Global suppression is considered a decrease in motor activity occurring in both a muscle whose action is being inhibited as well as in a muscle remote to the action being shut down. Corticospinal excitability is one way of providing a measure of motor output from the brain to muscles in order to control movement and offers an outlook on whether suppression or initiation of the muscle is occurring. When an unexpected event occurs there is a shift in attention which causes a global shut-down of cognitive and motor processes (Wessel \& Aron, 2017). If the unexpected event is a sudden postural perturbation, this global shut down of cognitive and motor function could pose a delay or cause an inability to recover postural control. The previously presented scenario of the bus suddenly stopping with groceries or bags around the passenger's feet and the need to shut off the step and select a more appropriate response of fixed support or a handle bar is an example of this issue.

A postural threat context is time sensitive for making an appropriate reaction and demands the desired action to be performed at high speed. When there needs to be an override for more appropriate behavior or simply because an unexpected event has 
occurred, this response inhibition is initiated if the context demands a cessation of action. In scenarios involving a postural threat, balance recovery may require suppression of an automated action when an unexpected event occurs if the automated action poses a greater threat to balance. Previous studies have explored the principles of rapid cessation of movement in context using go/no go signals to press buttons on a keyboard (D. S. A., Majid et al., 2012). Majid et al. (Majid et al., 2012) determined the brain used a suppression response to quickly suppress all muscles of the body, even those irrelevant to the task when they needed to quickly stop the action (in this instance, a leg muscle). When attempting to continue a motion, it has been found that the action desired to continue was extremely delayed due to global generic suppression (Majid et al., 2012). Global suppression of the motor system is thought to follow a hyper direct pathway between the frontal cortex and subthalamic nucleus of the basal ganglia by directly engaging to send inhibitory output decreasing the excitation of the motor output (Duque et al., 2017). Further investigation is necessary to fully understand the phenomina of whether global suppression is occurring in a postural threat context and the duration of this response inhibition. Balance control is an important factor affected by global suppression due to the increased risk of injury if balance is not successfully recovered by either shutting down an action or being able to initiate another action.

\section{Transcranial Magnetic Stimulation and motor evoked potentials}

In this study, Transcranial Magnetic Stimulation (TMS) was used to probe the excitatory state of the motor system. TMS is a noninvasive technique that can produce small electrical pulses that induce or inhibit activity in the human cortex (Klomjai, Katz, 
\& Lackmy-Vallée, 2015). When applied over the motor cortex, TMS activates interneurons, which in turn sends descending volleys to the spinal motor neurons. The spinal motor neurons are able to initiate an action potential sent to the targeted muscle and causes a muscle action (Klomjai, Katz, \& Lackmy-Vallée, 2015). When electromyography (EMG) sensors are placed over the muscle belly of targeted muscles, we are able to read the motor evoked potentials (MEPs) induced by the TMS. This can be used to observe response inhibition in a postural context to provide insight on the timing of inhibition. These inhibitory modes have been dissociated in seated tasks involving arbitrary stimulus-response relations, with the aid of transcranial magnetic stimulation (TMS) and EMG (Aron \& Verbruggen, 2008; Majid et al., 2012). TMS can be applied over the primary motor cortex (M1) to cause motor evoked potentials in targeted contralateral muscles, which can be recorded through surface EMG sensors (Wessel \& Aron, 2017). These MEPs will be able to measure the corticospinal excitability in these muscles. This measure will provide an outlook on whether specific muscle representations are facilitated or suppressed and how quickly this change in excitability occurs. The value of utilizing TMS is to allow us to assess the changes in motor output by corticospinal excitability fluctuations.

The purpose of this study is to determine if motor suppression occurs within a postural recovery context in muscles irrelevant to the task at hand.

Research hypothesis

When faced with an infrequent cue to suppress a forward step (unexpected event) we will see attenuation of the hand motor activity (irrelevant effector). This can 
reflect global suppression across the motor system to an unexpected event that requires rapid inhibition of a highly automatic (prepotent) action.

\section{Methods}

\section{Participants}

A total of 20 participants between the ages of 18-30 years old were recruited from a convenience sample from the population of students at Utah State University. Handedness was noted using the Edinburgh Handedness Inventory (Oldfield, 1971). All participants signed written informed consent in accordance with the Institutional Review Board of Utah State University. The participants were screened prior to testing to determine their suitability for TMS testing using guidelines developed by a group of experts (Rossi, Hallett, Rossini, \& Pascual-Leone, 2009). Particpants were excluded from the study based on responses to this screening.

\section{Data acquisition}

Electromyography (EMG) recordings were obtained using Delsys DE-2.1 differential surface electrodes, which contained preamplifiers sealed in polycarbonate enclosures (Delsys Inc., Boston, MA, USA). The electrode configuration included 2 silver bars each $10 \mathrm{~mm}$ long by $1 \mathrm{~mm}$ in width. Placement of EMG electrodes are indicated in Figure 3 by blue markings. EMG signals were amplified (gain =1000) using a Delsys Bagnoli-4 amplifier (Delsys Inc., Boston, MA, USA). Data was acquired and bandpass filtered $(10-1000 \mathrm{~Hz})$ using Signal Software and a Cambridge Electronic device (Power 1401, Cambridge Electronic Design, Cambridge, UK). Figure 1 illustrates 
the electromyogram produced pre-perturbation and post-perturbation. The muscles being tested in this study were selected based on their relevance for the stepping task as well as two task-irrelevant muscles in the hand. EMG recordings were collected for ankle dorsiflexors in both legs, the Tibialis Anterior (TA) muscles, and two intrinsic hand muscles in the right hand, the Opponens Pollicis (OP) and First Dorsal Interosseus (FDI). FDI response was the main focus to best answer the research question and OP provided information on hand grip response onset. The TA muscles of both legs were measured to detect stepping responses in either leg throughout testing used in conjunction with footswitches implanted in shoe insoles. A ground electrode was placed over the olecranon process of the right arm.

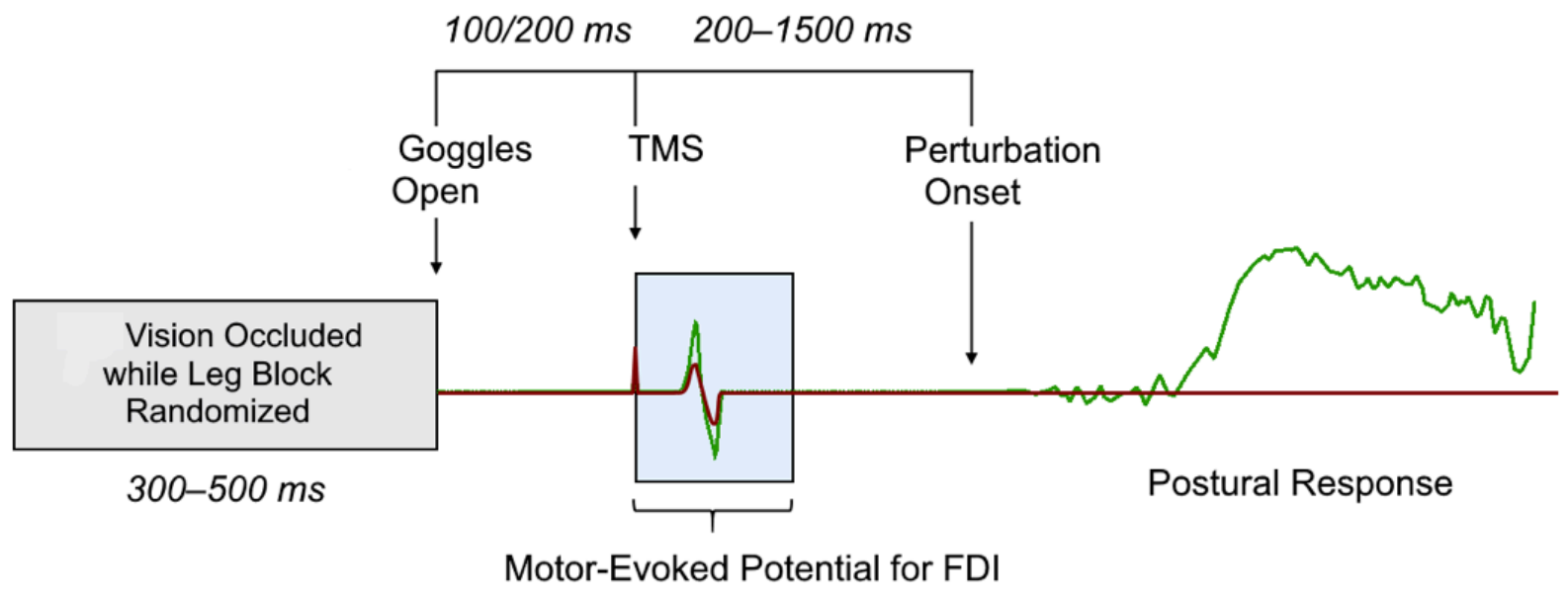

Figure 1. Trial sequence illustrated with fictitious electromyogram from first dorsal interosseous muscle pre-perturbation and tibialis anterior muscles post-perturbation.

\section{Testing Apparatus: Lean and Release}

A lean and release cable system was built to create unpredictable forward perturbations, pictured in Figure 3. The lean and release device has been successfully used in healthy adult populations as well as in clinical populations, as referenced in 
Figure $2 a$ to assess reactive balance (Lakhani, Mansfield, Inness, \& Mcllroy, 2011; Mansfield et al., 2011; Mansfield, Inness, Lakhani, \& Mcllroy, 2012). The direction and amount of perturbation were predictable to the participant, however during which trials and the onset the perturbations occurred was unpredictable to the participants.

All trials were conducted with the participant in a forward lean position of about $\sim 6$ degrees maintained in the body harness. The exact forward lean position for each participant was determined as the minimal lean angle where a change-of-support reaction (i.e. forward step) is necessary to recover balance with perturbation. The body harness was attached to a cable, which was secured to the wall behind the participant by a magnet that can release the cable to cause the sudden postural perturbation. The cable was attached to the posterior portion of the harness at the thoracic level. The participant was also secured by support cables attached to girders in the ceiling to prevent the participant from falling to the ground. The participant maintained the same position for the start of each trial, with feet shoulder width apart, leaning with hips forward, heels in contact with the ground, and relaxed. The participant was instructed to only react in the case of a perturbation, and to fix their gaze forward to where they are just able to see the leg block at the bottom of their line of vision when it is present. 


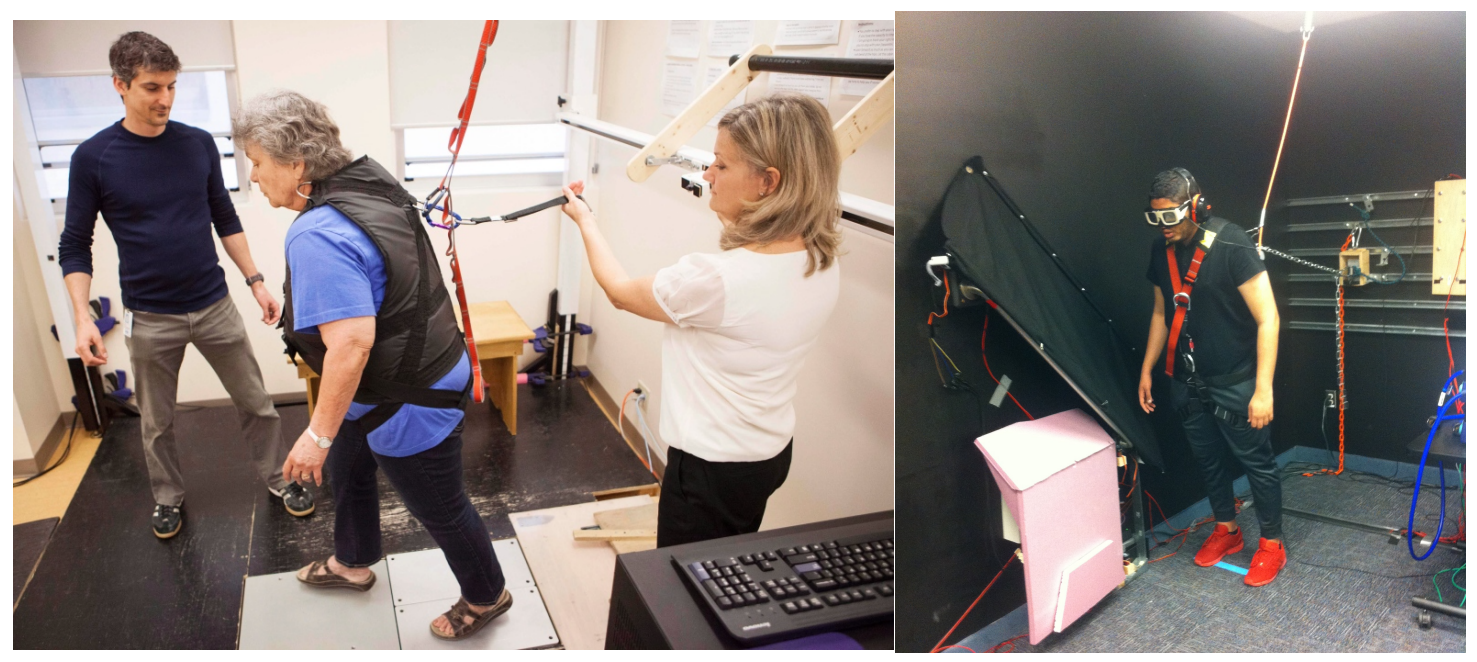

Figure 2. Left: depicts a lean and release system in a clinical setting. Right: The current study used a similar method with key changes. Vision was manipulated using occlusion goggles. Furthermore, the response environment was altered to either provide an open stepping path or impose a leg block. This allowed us to investigate how quickly and efficiently a participant could adapt their balance reactions to match the movement options afforded by the environment.

\section{Affordances and constraints}

A foam leg block was present directly in front of the participant to obstruct any stepping reactions during a few trials. In this case, the participant needed to maintain a fixed support to control balance during a perturbation. The leg block was intended to impede any unwanted step reaction; however, the leg block was not rigidly fixed and could be displaced by leg contact in the event of an unwanted reaction or if the participant was unable to avoid stepping in order to prevent injury with contact of the obstacle. When the leg block was not present, the participant was free to take a rapid forward step when a perturbation occurs.

\section{Control of visual cuing}

Visual access to the environment presented in front of the participant was limited to right before postural perturbation occurs. Access to vision was manipulated in this 
study by use of liquid crystal goggles (Translucent Technologies Inc. Toronto, ON, Canada). These goggles were programed to open $\sim 600 \mathrm{~ms}$ before postural perturbation. While closed, the scene in front of the participant changed to set up the configuration of obstacles for each trial. The participant was unaware of the setting until visual access is provided. Once the goggles opened, the participant had to quickly observe and adapt to the environment in front of them. The leg block was moved into position via computertriggered servo motors at the start of each trial regardless of condition to create consistent sound throughout testing. In addition to ear plugs and obscured vision, this was intended to avoid any advanced cueing for the upcoming condition.

\section{TMS Protocol}

In this study, TMS was delivered with a Magstim 200 (monophasic waveform) stimulator (Magstim Company Ltd., Whitland, UK) using a figure eight D70² Coil (Double 70 $\mathrm{mm}^{2}$ Coil - Magstim Company Ltd., Whitland, UK). A single pulse was delivered at two post-vision time points to the left primary motor cortex (M1), as illustrated in Figure 1. Markings were made on the scalp in order to locate the optimal area to stimulate, referred to as the hotspot, which was found while the patient was seated. Stimulating over this area excited a motor evoked potential (MEP) in the contralateral hand, specifically the right FDI. The hotspot was confirmed once the patient was standing and in the leaning position and here the intensity of the testing stimulus was determined. The optimal stimulus intensity provoked 1-1.5mV MEPs peak-to-peak in the right FDI. The TMS coil was maintained on the hotspot throughout the trials, as pictured in Figure 3, and the position was reset after any corrective balance response. The TMS sent a pulse during two time points $-100 \mathrm{~ms}, 200 \mathrm{~ms}$ - occurring after visual access. In 
previous studies performed in a non-postural threatening context, global suppression was seen to occur in task irrelevant muscles around 200ms (D. S. A. Majid et al., 2012). Due to the urgency to avoid the postural threat in this study, $100 \mathrm{~ms}$ was included in the event that the global suppression occurs sooner.

\section{Experimental Design}

After determining the TMS hotspot and intensity and the optimal lean angle, the participant went through several practice trials. They practiced remaining relaxed and only reacting in the case of a perturbation with a fixed support or change of support reaction. This familiarized them with the task prior to testing. During testing, the participants reacted only in the event of the cable release with a forward step (no leg block present) or by maintaining a fixed support (leg block was present). There were 6 testing blocks, each consisting of 26 trials of 13 possible conditions. This included trials that do not present visual access to the participant to normalize the MEP data to develop a baseline reference to account for any heightened corticospinal excitability related to any task related changes. About $75 \%$ of the trials required a forward recovery step with no leg block present. The other $25 \%$ of trials required a fixed support reaction with the leg block present. This uneven split for step/no step reactions was used to create a bias to the stepping action, ensuring this was the automated response, and making the no step task more unexpected and difficult. Perturbations via cable release only occurred on about 1/3 trials, at $\sim 600 \mathrm{~ms}$ post-vision. The other $2 / 3$ trials consisted of the participant remaining relaxed and simply observing the environment in front of them or remaining still with no visual access. This also allowed the perturbations to become more unexpected. 


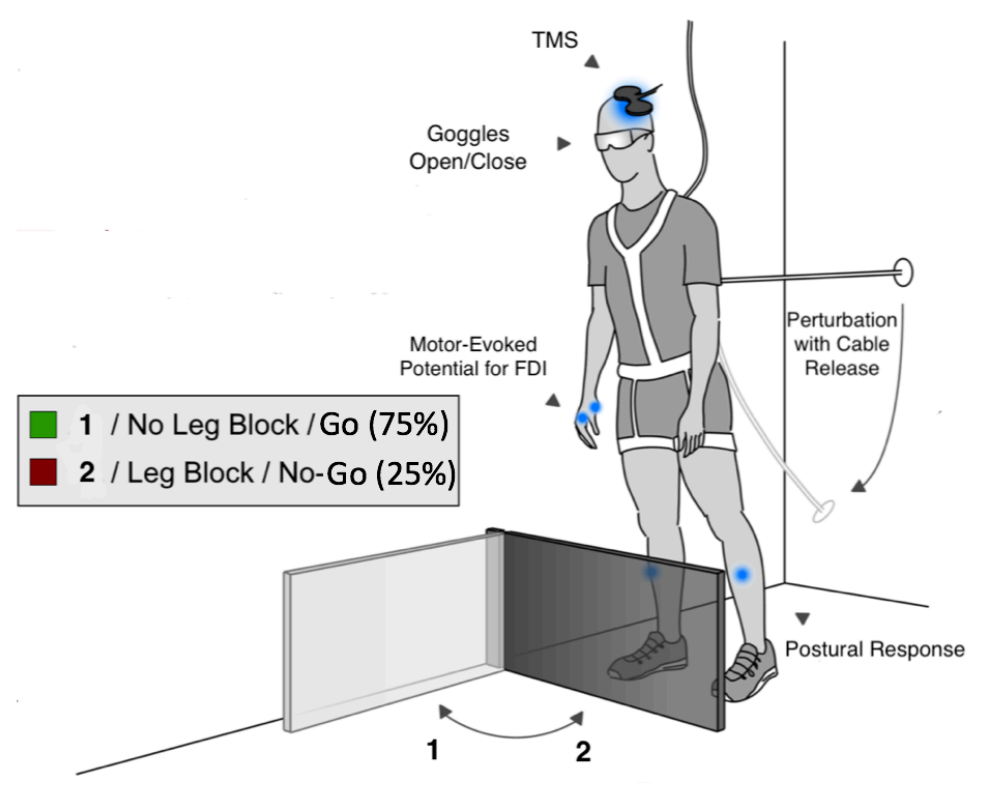

Figure 3. Illustrated is a schematic of the electrophysiological sites, lean-and-release apparatus, TMS site, liquid crystal goggles, and leg block/no leg block conditions.

\section{Data Analysis}

Data processing: Background EMG was determined from the root mean square of EMG activity for the FDI muscle in a time window of $100 \mathrm{~ms}$ immediately prior to TMS onset. If background EMG in this time window exceeded $10 \mu \mathrm{V}$, the trial was discarded. Moreover, any trials producing a very small MEP amplitude (i.e. $<100 \mu \mathrm{V}$ peak-to-peak) were excluded. As a final step, outliers were identified as those values falling outside the threshold defined by 1.5 times the interquartile range, and these outliers will also be excluded.

MEP amplitude was determined as the peak-to-peak amplitude from approximately $15 \mathrm{~ms}$ to $50 \mathrm{~ms}$ post TMS. To help standardize data, average MEP amplitudes were converted into z-scores to reduce potential variability between test blocks within an individual and to reduce inter-subject variability (Hasbroucq et al., 
1999; Klein-Flügge \& Bestmann, 2012). The mean and standard deviations of the MEP amplitudes during 'No-Vision' trials for each test block were used as a reference, for each participant separately. The individual MEP amplitudes observed in the other two conditions (step, no-step) were converted into z-scores calculated from this reference. These normalized values were then grouped for statistical analysis.

Statistical analysis: A 2 × 2 repeated measures ANOVA was used to test for interactions between factors 'Condition' (step/no-step) and 'Time' (100ms, 200ms) for the MEP amplitude in the FDI muscle. In this study, only leg reactions were relevant to avoid a fall but the main outcome measure here was hand muscle corticospinal excitability to determine global suppression occurrence when participants were prevented from using a prepotent recovery step (i.e. force to use a fixed support reaction). To test our specific hypothesis that MEPs from a hand muscle (FDI) would be suppressed when participants were forced to stop an automated forward step, directional paired t-tests were used to compare MEP amplitude at the 200ms delay versus the 100ms (baseline) delay for the STEP and NO-STEP conditions $(\alpha<0.05)$.

\section{Results}

As predicted, suppression of the FDI hand musculature was suppressed during trials requiring inhibition of a forward step. Data was collected for twenty subjects but one dataset was discarded due to insufficient trials obtained. Age of subjects ranged from nineteen to twenty-five with two females and the remaining eighteen participants being male. The average TMS testing intensity was set to evoke an MEP amplitude of $1-1.5 \mathrm{mV}$ 
peak-to-peak. The time $\mathrm{x}$ step condition interaction was significant using a $2 \times 2$ repeated measures ANOVA $(F(1,18)=4.47, \mathrm{p}=0.049)$, as graphed in Figure 4. There was also a significant main effect for time $(F(1,18)=5.832, \mathrm{p}=0.027)$, which was driven by the large drop in MEP amplitude for no-step trials by 200ms post-vision. There was no main effect for condition $(F(1,18)=0.396, \mathrm{p}=0.537)$. Visual inspection of the line graph in Figure 4 reveals decreasing MEP amplitude over time for the NO-STEP condition only and this was confirmed with follow-up comparisons. Specifically, these comparisons revealed a significant decrease at $200 \mathrm{~ms}$ compared with $100 \mathrm{~ms} \mathrm{t}_{18}=2.595, \mathrm{p}=0.009$ for NO-STEP condition. By contrast a similar comparison between $200 \mathrm{~ms}$ and $100 \mathrm{~ms}$ for the STEP condition reveals no difference $\mathrm{t}_{18}=0.346, \mathrm{p}=0.367$.

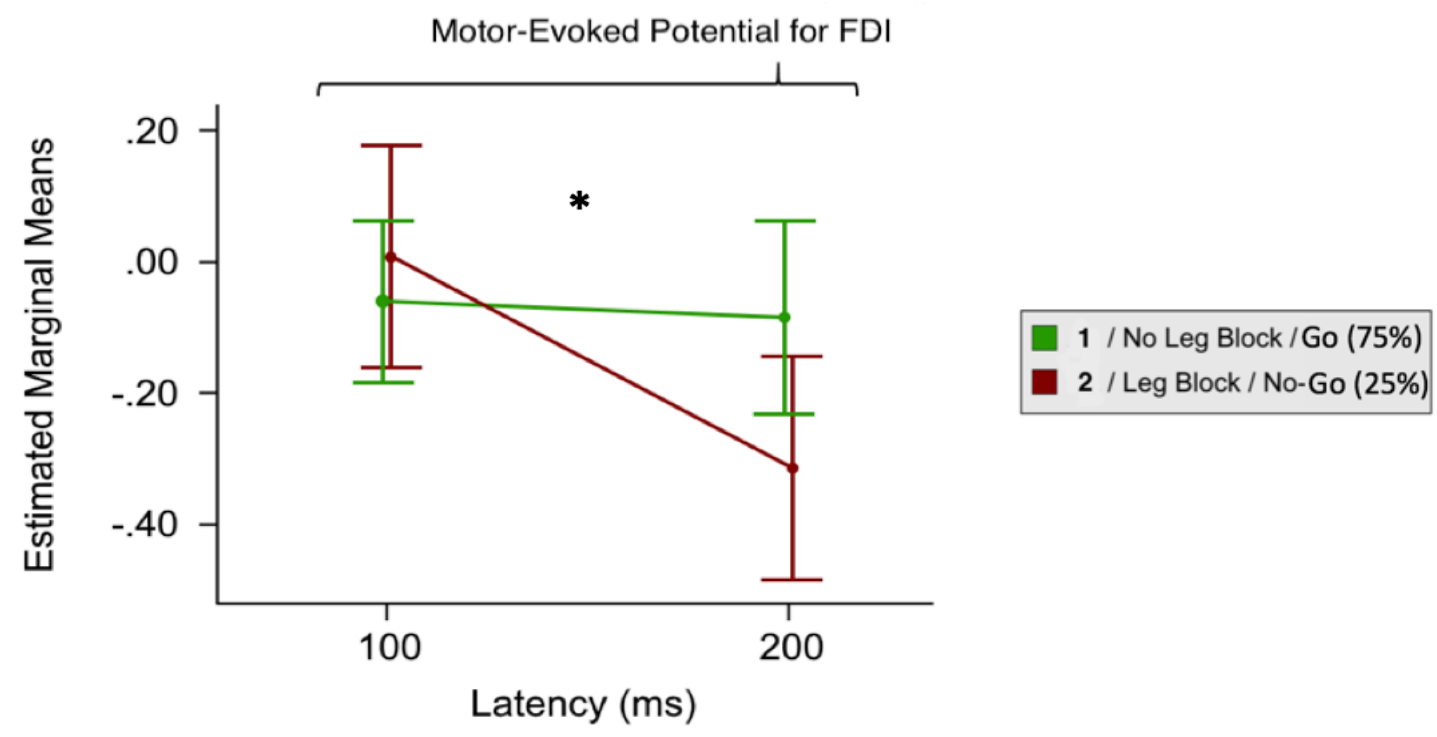

Figure 4. Graphed is the evidence for global suppression from the 2 x 2 repeatedmeasures ANOVA. Probabilities exclude two no-vision/no-release reference trials per 18 -trial block. Error bars demarcate \pm 1 standard error. ${ }^{*} p<.05$. 


\section{Discussion}

Corticospinal excitability was measured in the FDI musculature, a task irrelevant hand muscle, during tasks requiring either a forward step to recover balance when a postural perturbation occurs or occasional suppression of the recovery step. The experiment showed that inhibition of a recovery step also resulted in suppression of a task irrelevant hand muscle. This finding suggests that global suppression may have led to the shut down of a task-irrelevant hand muscle when emergently needing to suppress balance recovery step, confirming the hypothesis. Suppression of the hand musculature was observed at $200 \mathrm{~ms}$, but not at $100 \mathrm{~ms}$, which may indicate an early temporal limit for when this widespread suppression may impact motor output (i.e. somewhere between 100 and 200ms).

\section{Major findings in relation to current literature on global suppression}

The time of $200 \mathrm{~ms}$ was chosen due to a proven onset of global suppression in seated tasks at 200ms (Majid et al., 2012) whereas 100ms was chosen in the event that postural threat resulted in an earlier onset for this suppression. This current study extends from past research by demonstrating this widespread suppression effect on muscular activation in the hand while maintaining upright balance with the legs. Present findings are of particular significance given that the maintenance of balance is time sensitive due to muscle reaction time and the risk of injury with postural threat tasks compared to the seated tasks. The impending risk of injury if balance is not successfully recovered creates a greater demand for cognitive attention to the task at hand. Wessel and Aron (2017) stated that, "signal detection, inhibition, attention orienting, and the potential suppression 
of ongoing cognitive processes are all part of a tightly linked cascade of interacting neural processes." This portrays a general motor control process in that when an unexpected event occurs the brain stops all processes and cognitive thought and attention to reboot. This shut down allows for a restart to judge the appropriate action to make next.

Wessel and Aron (2017) clarified that there is impaired cognitive performance after unexpected events due to a change in attention. This affects postural recovery after stopping because of the timing and reset of action. Typically, the onset of TA muscles in response to perturbations takes an average of $82 \mathrm{~ms}$ in young individuals for onset of a step (Thelen et al., 2000). Initiation of a new action to recover balance following a prolonged period of muscle inhibition may make it difficult to prevent a fall due to sensitivity of time.

\section{Methodological considerations}

There were several limitations to this study that could be improved upon in future research. The scope of timing being investigated could be expanded to include times between 100-200ms and also further out to provide a better picture of the onset of motor suppression and the liberation of suppression. The times of $100 \mathrm{~ms}$ and $200 \mathrm{~ms}$ were investigated in this study due to $200 \mathrm{~ms}$ being shown to display global suppression in seated tasks (Majid et al., 2012) and 100ms since the study included a postural threat and the global suppression was considered to occur sooner in a postural threat context. By including a larger range of TMS probes we could better isolate the general timing of onset for global suppression in a postural threat context and determine when the body is 
able to initiate new action. The corticospinal excitability of other task muscles could have also been investigated to better confirm the concept of global suppression. Muscles such as the trunk, upper leg, and hip may also play a role in reacting and preventing a fall and can also be investigated to determine if suppression occurs in task relevant muscles as well as the task irrelevant muscles. The general set-up of lean-and-release apparatus could be improved upon to be more efficient and prevent more errors such as moving the apparatus away from the wall, which had a handle that was covered for other studies. This would prevent participants from attempting to grab onto the handle or wall for support despite the handle being covered. The stool used to stand and administer the TMS pulse was also in the view and reach of the participant. Throughout the study, many participants used the stool, the handle, and the wall to grab onto to prevent a fall rather than regaining balance with their legs or a step. The reliability of the leg block apparatus was also faulty due to mechanical limitations; there were many times that that the leg block moved partway to block the step. A more sustainable leg block could have been developed to prevent this issue.

\section{Future research}

Further research needs to investigate the time course of global suppression in a balance recovery context. This effect can have potentially important consequences when we are forced to initiate a new action to regain control of balance after suppressing an automated action (e.g. avoiding clutter on a bus by shutting off a step into the object and initiating a new action of grabbing the handrail or changing direction of the step around the object). The speed at which this suppression emerges and how long it lasts can be 
crucial in the time pressured world of balance control. A widespread motor suppression may delay action reprogramming, ultimately leaving an individual vulnerable to a fall.

Investigation should be performed looking into the specific cognitive processes of response inhibition to determine how flexible the brain is between two methods of response inhibition - global suppression, as presently studied, versus selective suppression, which as the name implies allows some actions to occur while suppressing others. There is a greater ability to selectively stop actions when there is foreknowledge of what actions need to be suppressed (Claffey, Sheldon, Stinear, Verbruggen, \& Aron, 2010). In situations where response inhibition is initiated, any advanced knowledge of the appropriate action needed - such as availability of a handrail on a ramp or stairs- or knowledge of what action needs to be turned off and which needs to be turned on can influence whether selective inhibition is initiated. If advanced knowledge is critical to selective inhibition, it is unclear how selective inhibition comes into effect in unpredictable environments and the maintenance of control of balance and it needs further investigation.

Global suppression in a postural threat context should also be explored further in an elderly population to determine age-related cognitive deterioration relating to balance and muscle suppression. It is shown that the rate of falls is increased in older adults and relates to resulting injuries (Bergen et al., 2016). Since reactive balance requires higher brain functioning, it would be helpful to determine what brain processes are most effected by deterioration in the elderly population. Elderly participants have exhibited a decreased capacity to generate muscle inhibition (Bönstrup, Hagemann, Gerloff, Sauseng, \& Hummel, 2015). This could provide more insight into the mechanism linking cognitive 
decline and falls. Rehabilitation protocols addressing global inhibition, or simply response inhibition, could provide a clinical impact by attending to this area of deficit in balance control with aging.

\section{Conclusions}

In conclusion, this current study was able to confirm the presence of suppression of muscle activity in a postural threat context occurring in a task-irrelevant intrinsic hand muscle (FDI). This finding provides support for a global suppression mechanism. This study was a beneficial extension of the investigation of global suppression because it included a balance control component, which needs to be understood due to the risk of fatal and nonfatal injuries due to falls. This study provided a strong basis to investigate global suppression and when it occurs in a postural threat and balance recovery context. 


\section{References}

Aron, A. R., \& Verbruggen, F. (2008). Stop the Presses: Dissociating a Selective From a Global Mechanism for Stopping. Psychological Science, 19(11), 1146-1153. https://doi.org/10.1111/j.1467-9280.2008.02216.x

Bergen, G., Stevens, M. R., \& Burns, E. R. (2016). Falls and Fall Injuries Among Adults Aged $\geq 65$ Years — United States, 2014. MMWR. Morbidity and Mortality Weekly Report, 65(37), 993-998. https://doi.org/10.15585/mmwr.mm6537a2

Bolton, D. A. (2015). The role of the cerebral cortex in postural responses to externally induced perturbations. Neuroscience and Biobehavioral Reviews, 57(Journal Article), 142-155. https://doi.org/S0149-7634(15)00232-8 [pii]

Bönstrup, M., Hagemann, J., Gerloff, C., Sauseng, P., \& Hummel, F. C. (2015). Alpha oscillatory correlates of motor inhibition in the aged brain. Frontiers in Aging Neuroscience, 7. https://doi.org/10.3389/fnagi.2015.00193

Claffey, M. P., Sheldon, S., Stinear, C. M., Verbruggen, F., \& Aron, A. R. (2010). Having a goal to stop action is associated with advance control of specific motor representations. Neuropsychologia, 48(2), 541-548.

https://doi.org/10.1016/j.neuropsychologia.2009.10.015

Duque, J., Greenhouse, I., Labruna, L., \& Ivry, R. B. (2017). Physiological Markers of Motor Inhibition during Human Behavior. Trends in Neurosciences, 40(4), 219236. https://doi.org/10.1016/j.tins.2017.02.006

Hasbroucq, T., Osman, A., Possamaï, C.-A., Burle, B., Carron, S., Dépy, D., ... Mouret, I. (1999). Cortico-spinal inhibition reflects time but not event preparation: neural 
mechanisms of preparation dissociated by transcranial magnetic stimulation. Acta Psychologica, 101(2), 243-266. https://doi.org/10.1016/S0001-6918(99)00007-4

Herman, T., Mirelman, A., Giladi, N., Schweiger, A., \& Hausdorff, J. M. (2010). Executive control deficits as a prodrome to falls in healthy older adults: a prospective study linking thinking, walking, and falling. The Journals of Gerontology.Series A, Biological Sciences and Medical Sciences, 65(10), 10861092. https://doi.org/10.1093/gerona/glq077

Klein-Flügge, M. C., \& Bestmann, S. (2012). Time-Dependent Changes in Human Corticospinal excitability Reveal Value-Based Competition for Action during Decision Processing. Journal of Neuroscience, 32(24), 8373-8382. https://doi.org/10.1523/JNEUROSCI.0270-12.2012

Klomjai, W., Katz, R., \& Lackmy-Vallée, A. (2015). Basic principles of transcranial magnetic stimulation (TMS) and repetitive TMS (rTMS). Annals of Physical and Rehabilitation Medicine, 58(4), 208-213.

https://doi.org/10.1016/j.rehab.2015.05.005

Lakhani, B., Mansfield, A., Inness, E. L., \& Mcllroy, W. E. (2011). Characterizing the determinants of limb preference for compensatory stepping in healthy young adults. Gait \& Posture, 33(2), 200-204. https://doi.org/10.1016/j.gaitpost.2010.11.005

Majid, D. S. A., Cai, W., George, J. S., Verbruggen, F., \& Aron, A. R. (2012). Transcranial Magnetic Stimulation Reveals Dissociable Mechanisms for Global Versus Selective Corticomotor Suppression Underlying the Stopping of Action. Cerebral Cortex, 22(2), 363-371. https://doi.org/10.1093/cercor/bhr112 
Maki, B. E., \& McIlroy, W. E. (2007). Cognitive demands and cortical control of human balance-recovery reactions. Journal of Neural Transmission, 114(10), 1279-1296. https://doi.org/10.1007/s00702-007-0764-y

Mansfield, A., Inness, E. L., Komar, J., Biasin, L., Brunton, K., Lakhani, B., \& McIlroy, W. E. (2011). Training rapid stepping responses in an individual with stroke. Physical Therapy, 91(6), 958-969. https://doi.org/10.2522/ptj.20100212

Mansfield, A., Inness, E. L., Lakhani, B., \& McIlroy, W. E. (2012). Determinants of limb preference for initiating compensatory stepping poststroke. Archives of Physical Medicine and Rehabilitation, 93(7), 1179-1184.

https://doi.org/10.1016/j.apmr.2012.02.006

Mirelman, A., Herman, T., Brozgol, M., Dorfman, M., Sprecher, E., Schweiger, A., ... Hausdorff, J. M. (2012). Executive function and falls in older adults: new findings from a five-year prospective study link fall risk to cognition. PloS One, 7(6), e40297. https://doi.org/10.1371/journal.pone.0040297

Muir, S. W., Gopaul, K., \& Montero Odasso, M. M. (2012). The role of cognitive impairment in fall risk among older adults: a systematic review and meta-analysis. Age and Ageing, 41(3), 299-308. https://doi.org/10.1093/ageing/afs012

Oldfield, R. C. (1971). The assessment and analysis of handedness: the Edinburgh inventory. Neuropsychologia, 9(1), 97-113.

Rogers, M. W., \& Mille, M.-L. (2016). Timing paradox of stepping and falls in ageing: not so quick and quick(er) on the trigger: Protective stepping and falls in ageing. The Journal of Physiology, 594(16), 4537-4547. https://doi.org/10.1113/JP271167 
Rossi, S., Hallett, M., Rossini, P. M., \& Pascual-Leone, A. (2009). Safety, ethical considerations, and application guidelines for the use of transcranial magnetic stimulation in clinical practice and research. Clinical Neurophysiology, 120(12), 2008-2039. https://doi.org/10.1016/j.clinph.2009.08.016

Thelen, D. ., Muriuki, M., James, J., Schultz, A. ., Ashton-Miller, J. ., \& Alexander, N. . (2000). Muscle activities used by young and old adults when stepping to regain balance during a forward fall. Journal of Electromyography and Kinesiology, 10(2), 93-101. https://doi.org/10.1016/S1050-6411(99)00028-0

Wessel, J. R., \& Aron, A. R. (2017). On the Globality of Motor Suppression: Unexpected Events and Their Influence on Behavior and Cognition. Neuron, 93(2), 259-280. https://doi.org/10.1016/j.neuron.2016.12.013 